Open Access

\title{
Successful Treatment of Duodenal Variceal Bleeding by Endoscopic Clipping
}

\author{
Su Bin Park, Sang Ho Lee, Jin Hee Kim, Hyun Jung Lee, Sung Pil Jang, Jae Nam Lee and Jong Ho Hwang \\ Department of Internal Medicine, Busan Medical Center, Busan, Korea
}

\begin{abstract}
Duodenal varix bleeding is an uncommon cause of gastrointestinal bleeding in patients with portal hypertension but can cause severe and potentially fatal bleeding. However, the incidence is low and a good treatment method has not been well established yet. Duodenal variceal bleeding can be treated surgically or nonsurgically. We have successfully treated a patient with duodenal variceal bleeding secondary to liver cirrhosis using hemoclips to control the bleeding.
\end{abstract}

Key Words: Gastrointestinal bleeding; Duodenal varix; Endoscopic clipping

\section{INTRODUCTION}

Variceal bleeding is a common cause of upper gastrointestinal bleeding in patients with liver cirrhosis. It happens as a massive bleeding and has a very high rate of rebleeding, requiring quick and accurate diagnosis and treatment. Variceal bleeding mainly occurs at the esophagus or the stomach, but can also rarely occur at the duodenum, jejunum, ileum, appendix, colon, rectum, and the biliary tract. Of these, duodenal variceal bleeding has a mortality of $40 \%$ and poor prognosis. ${ }^{1}$ Treatment of duodenal variceal bleeding includes endoscopic sclerotherapy, endoscopic ligation, and transjugular intrahepatic portosystemic shunt (TIPS). But because the incidence of duodenal variceal bleeding is low, the best treatment method has not yet been researched well. ${ }^{1}$ We have successfully treated a patient who was admitted with duodenal variceal bleeding using hemoclips endoscopically and report this case with a review of the literature.

\footnotetext{
Received: May 27, 2012 Revised: July 2, 2012

Accepted: July 3, 2012

Correspondence: Sang Ho Lee

Department of Internal Medicine, Busan Medical Center, 359 Worldcup-daero, Yeonje-gu, Busan 611-706, Korea

Tel: +82-51-507-3000, Fax: +82-51-607-2807, E-mail: skylsh@kornet.net

(c) This is an Open Access article distributed under the terms of the Creative Commons Attribution Non-Commercial License (http://creativecommons.org/ licenses/by-nc/3.0) which permits unrestricted non-commercial use, distribution, and reproduction in any medium, provided the original work is properly cited.
}

\section{CASE REPORT}

A 49-year-old male patient presented with hematemesis and hematochezia. He had been diagnosed with alcoholic liver cirrhosis 14 years ago, and had hyperthyroidism and diabetes.

At the time of admission the patient's blood pressure was $60 / 30 \mathrm{~mm} \mathrm{Hg}$, pulse rate was 122 per minute, and respiratory rate was 20 per minute. Basal body temperature was $36^{\circ} \mathrm{C}$ and blood glucose level was $257 \mathrm{mmol} / \mathrm{L}$. Physical examination showed acutely ill appearance with mild confusion. The patient had conjunctival pallor, no scleral icterus and no abnormalities on chest auscultation. The abdomen was soft to palpation but was distended, and there was tenderness to palpation at the epigastrium without rebound tenderness.

Laboratory findings showed hemoglobin concentration of $7.9 \mathrm{~g} / \mathrm{dL}$, leukocytes $12,060 / \mathrm{mm}^{3}$ (neutrophils $86.7 \%$ ), and platelets $101,000 / \mathrm{mm}^{3}$. Serum biochemistry showed the total protein concentration to be $4.9 \mathrm{~g} / \mathrm{dL}$, albumin concentration $2.0 \mathrm{~g} / \mathrm{dL}$, total bilirubin $0.52 \mathrm{mg} / \mathrm{dL}$, blood urea nitrogen 15.4 $\mathrm{mg} / \mathrm{dL}$, creatinine $0.9 \mathrm{mg} / \mathrm{dL}$, aspartate aminotransferase/alanine aminotransferase 31/51, alkaline phosphatase $93 \mathrm{IU} / \mathrm{L}$, prothrombin time 14.4 seconds (international normalised ratio, 1.21), while all of hepatitis B surface antigen, hepatitis B surface antibody, and antihepatitis $C$ virus were negative.

An emergency upper gastrointestinal endoscopy was performed on the day of the patient's admission. It showed a varix (F3, Lm, Cb, RC-) without red color sign from all along the mid to lower esophagus. Apart from signs of congestive gas- 
tropathy, there were no other lesions suggestive of bleeding. Along the entire wall of the duodenal bulb was a pulsating blue dumbbell-shaped varix and stigma on the surface of this varix (Fig. 1). This was considered to be a bleeding focus, so the efferent vein and afferent vein were ligated using hemoclips, and hemostasis was achieved following hemoclips placement directly on the visible vessel (Fig. 2).

Three dimensional (3D) computed tomographic (CT) angiography was performed to verify the duodenal varix and the collateral pathways which showed a broad collateral varix around the duodenum and the head of the pancreas due to thrombosis of the main portal vein and the splenic vein (Fig. 3). Following 3D CT angiography, somatostatin intravenous

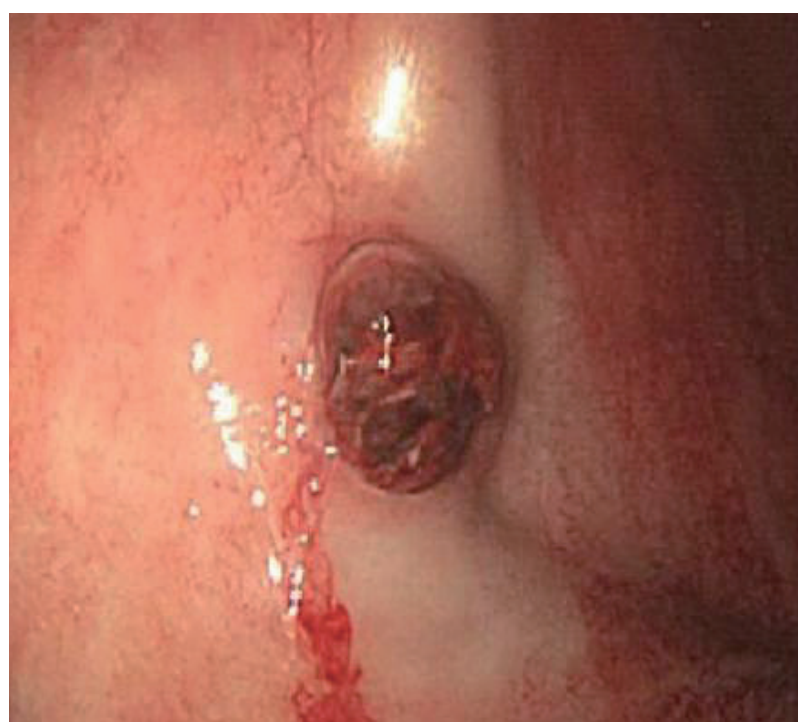

Fig. 1. Pulsating varix with hemorrhagic stigmata in the bulb of duodenum, an ulceration with exposed vessel on the varix.

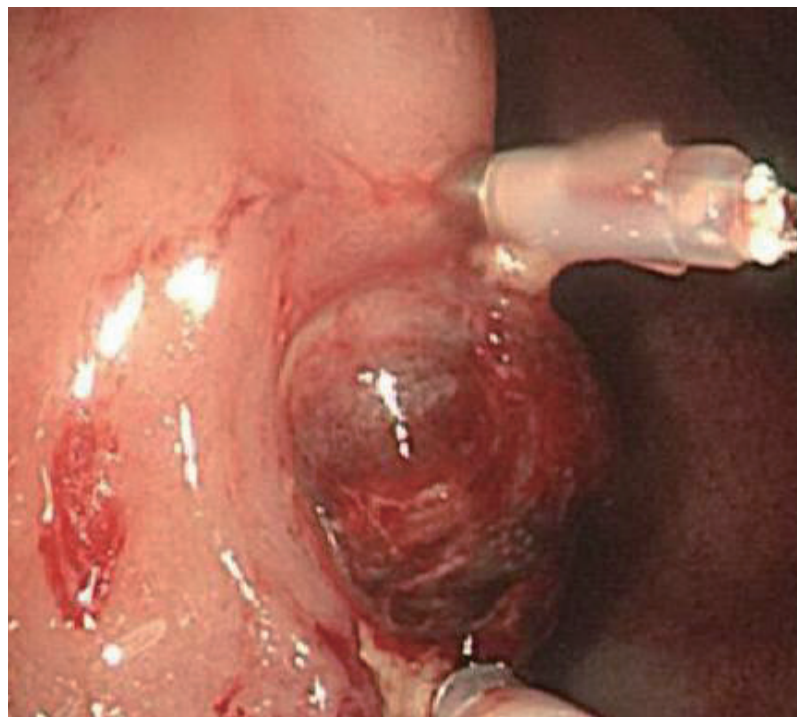

Fig. 2. Efferent vein and afferent vein were ligated using hemoclips to achieve hemostasis. injection and blood transfusion were given along with other conservative management. Although the patient had large amounts of melena, his vital signs were stabilized. A followup endoscopy 3 days after he procedure showed no signs of bleeding and two hemoclips were fixed on the varix (Fig. 4). The vital signs were stable, without any more melena, and the patient was discharged from the hospital 14 days after his first admission. Repeat endoscopy 3 months later revealed persis-

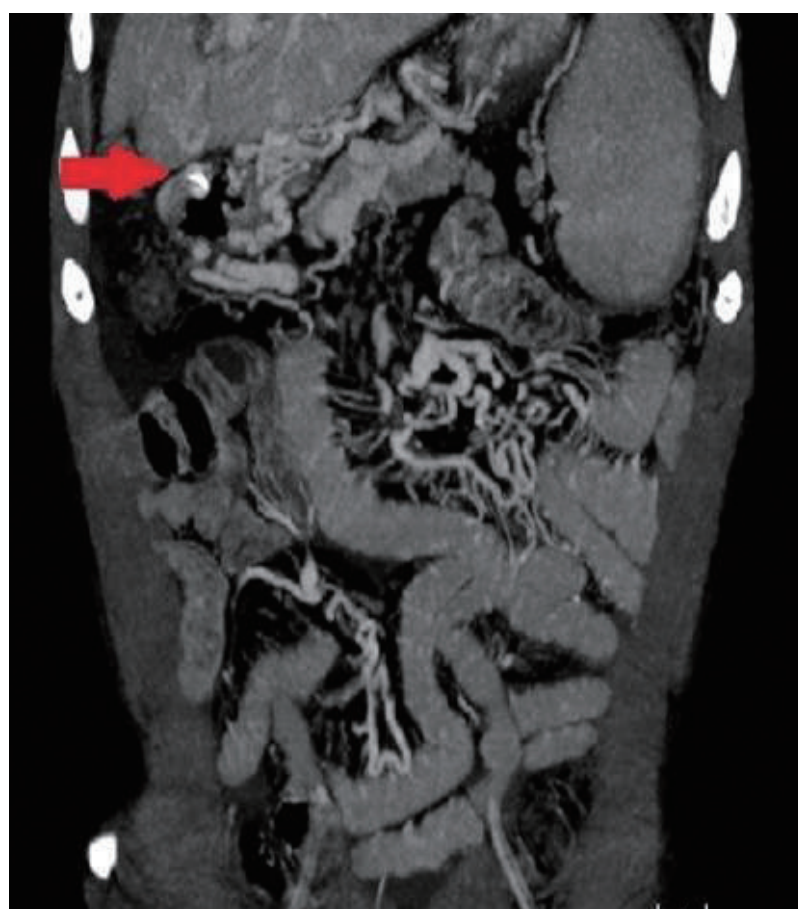

Fig. 3. Three dimensional computed tomographic angiography after treatment. Extensive collateral varix around the duodenum due to thrombosis in the main portal vein and splenic vein (arrow).

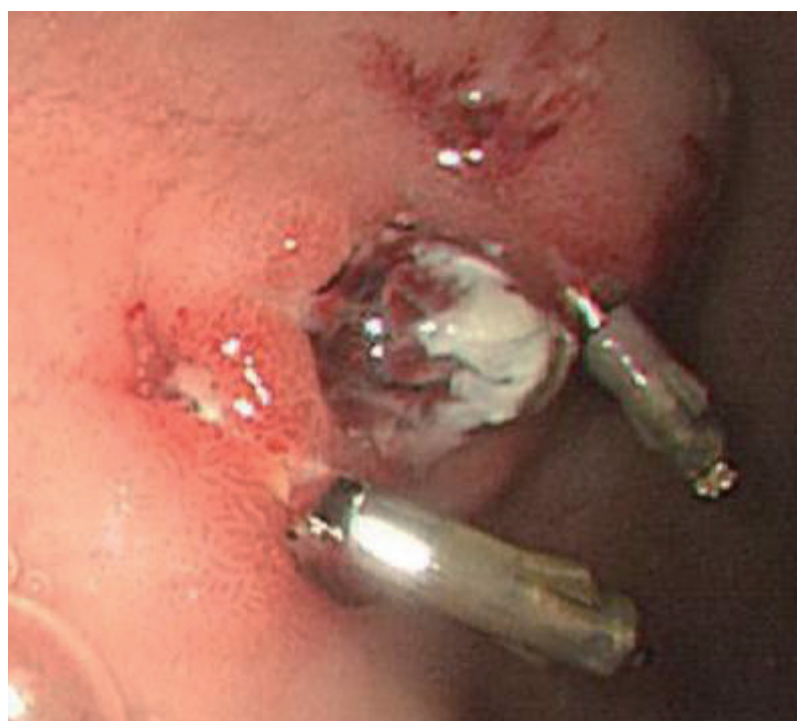

Fig. 4. Follow-up endoscopy 3 days after hemoclipping of the duodenal varix revealing a small ulcer. 


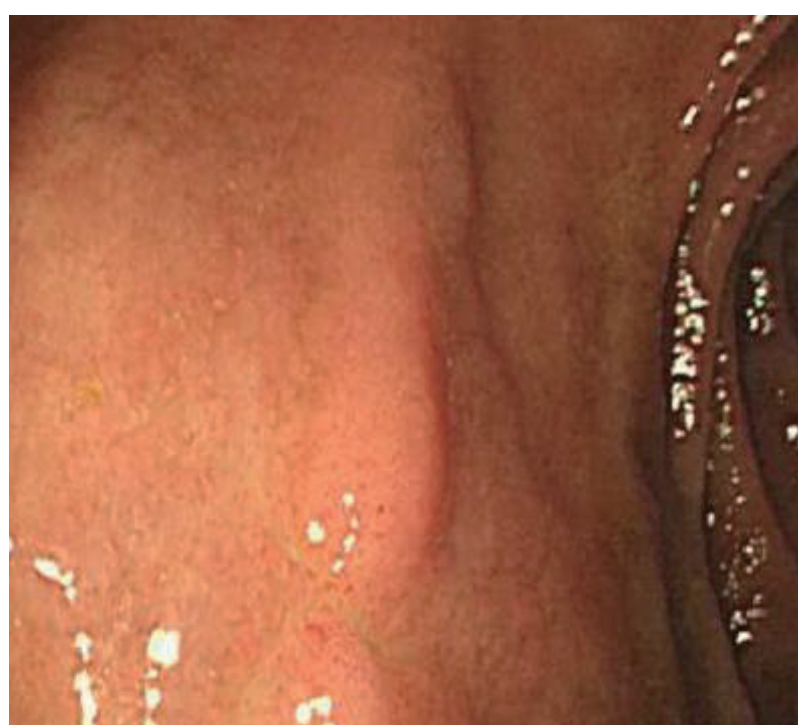

Fig. 5. Follow-up endoscopy 3 months after duodenal varix bleeding.

tent duodenal varix but of a reduced size and absence of the stigmata of recent bleeding (Fig. 5). Since then, the patient has not had any rebleeding episodes and is being followed up on an outpatient basis.

\section{DISCUSSION}

Duodenal varices are not as common as varices of the esophagus or the stomach. The prevalence of duodenal varices is related to the cause of portal hypertension and the technique used to show the varices; Up to $40 \%$ of patients with portal hypertension undergoing angiography showed duodenal varices. ${ }^{1}$ Although the frequency of bleeding is low, when it does bleed, it can be fatal with a mortality of $35 \%$ to $40 \% .{ }^{2}$ The most common site of occurrence is at the duodenal bulb and the frequency of occurrence decreases as the site is closer to the distal duodenum. This is because duodenal varices are located deep in the serosal layer, while esophageal varices are located in the submucosal area. ${ }^{3}$ The most common cause (30\%) of duodenal varices is portal hypertension due to liver cirrhosis. Other causes include occlusion of the splenic vein due to pancreatitis, tumors or thrombosis (25\%), and occlusion of the portal vein due to thrombosis, infection, and tumors $(25 \%)^{4}$

The pathogenesis is due to the increasing portal venous pressure which causes portosystemic communication. Duodenal varices are one of the ectopic varices caused by retroperitoneal portosystemic shunts. This is due to the increase in hepatofugal blood flow through the cystic branches of the pyloric veins, gastroduodenal veins, and the superior and inferior pancreaticoduodenal veins. ${ }^{5}$

Duodenal variceal bleeding is mainly diagnosed from en- doscopic examination of the upper gastrointestinal system. However, it is difficult to visualize by using endoscopies if they are located in the distal or lower part of the second part of the duodenum. If the site of bleeding cannot be identified using endoscopic methods, other methods can be considered, such as abdominal CTs, mesenteric angiography and explorative laparotomy.

Because of the infrequency with which bleeding duodenal varices can be found, there have been no randomized trials on the management of this condition, and the treatment guidelines have not been established. Therefore, management depends on local expertise and the cause of portal hypertension. ${ }^{1}$ In the past, surgical treatment methods such as variceal ligation, variceal excision, splenorenal shunting and partial duodenectomy were used, but the rate of postsurgical mortality was as high as $30 \%$ and such methods are not commonly used in recent years. ${ }^{2}$ Medical treatment of duodenal variceal bleeding is often limited. Recently, sclerotherapy, endoscopic ligation and radiological intervention procedures as well as TIPS and balloon-occluded retrograde transvenous obliteration have been used for treatments.

Emergency endoscopic sclerotherapy is useful as a first-line therapeutic measure in treating duodenal variceal bleeding. ${ }^{1}$ Several studies have reported the use of various types of sclerosant agent, including ethanolamine, polidocanol, dextrose $50 \%$ solution with $3 \%$ sodium tetradecylsulfate and polidocanol/thrombin. ${ }^{67}$ Sclerotherapy has been performed for the management of duodenal variceal bleeding and suggested as an effective measure. However, it carries a risk of perforation, embolism, and tissue injury. ${ }^{8}$

Endoscopic variceal ligation can also be a treatment method, but it has been reported that its use is limited in cases of severe bleeding because it is difficult to obtain a good viewing field and accessibility to collateral vessels is poor. ${ }^{2,9}$ Several authors have also questioned the safety of endoscopic variceal ligation in treating large varices. This is because if a varix is large, it is difficult to band the whole varix and large defects can occur when the band is removed. ${ }^{1}$

TIPS is a relatively safer treatment method and is used in patients who have poor liver function or if the endoscopic accessibility is limited due to severe bleeding. There have been cases where TIPS was used alone, or as a second-line therapy when endoscopic treatment methods have failed. ${ }^{10}$ TIPS can be used in patients with severe liver dysfunction, but its use is limited in patients with severe liver atrophy and can also increase the risk of developing complications such as hepatic encephalopathy or cerebral embolization. ${ }^{11,12}$

We have diagnosed duodenal variceal bleeding endoscopically in a patient with liver cirrhosis who was admitted to the hospital for gastrointestinal bleeding. We have successfully 
treated the bleeding using hemoclips at the afferent and efferent parts of the varix together. The bleeding can be immediately stopped if hemoclips are used to occlude both ends of the bleeding vessel accurately. Endoscopic sclerotherapy is used as the standard treatment, but repetitive sclerotherapy increases the risk of perforation due to thin wall of the duodenum. Moreover, the varix in this case was located on the duodenal bulb, anterior wall. There was not enough space to perform the procedure, and the varix would not have been sucked into the scope well. The advantage of endoscopic clipping is that it can directly occlude the bleeding vessel in patients who also has coagulopathy. Hemoclips are designed as an alternative treatment to control bleeding by direct mechanical pressure. Also, because hemoclips are made of steel, it is not removed by inflammatory reactions. Hemoclips generally fall off after 10 to 14 days of clipping, after the lesion has partly healed and when it is expected not to rebleed. ${ }^{13}$ Yol et al. ${ }^{14}$ reported that the effectiveness of endoscopic clipping in the hemostasis of bleeding esophageal varices could be compared with that of band ligation. Considering the advantage of high initial hemoastasis rate and decreased risk of bleeding, they suggested that endoscopic clipping is more effective than band ligation. Morimoto et al. ${ }^{15}$ have also reported a case where active gastric variceal bleeding was treated using metallic clips.

However, our approach should be used cautiously and reconsidered for the application of clipping. This clipping method has some potential to perforate the duodenum and to aggravate bleeding if misplaced; so endoscopic clipping therapy can be an option for variceal bleeding, not for general situation. And we could suspect the path of varix with gross finding of linear elevated change around the exposed vessel in this patient.

Duodenal variceal bleeding is not a common cause of gastrointestinal bleeding. Therefore, if not evaluated carefully, the treatment and management can be delayed, causing life-threatening massive bleeding. It is important to always consider the possibility of duodenal variceal bleeding in patients with portal hypertension and gastrointestinal bleeding. Although an effective treatment method has not been established yet, successful management of duodenal variceal bleeding can be expected by using a combination of endoscopic clipping.

\section{Conflicts of Interest}

The authors have no financial conflicts of interest.

\section{REFERENCES}

1. Norton ID, Andrews JC, Kamath PS. Management of ectopic varices. Hepatology 1998;28:1154-1158.

2. Khouqeer F, Morrow C, Jordan P. Duodenal varices as a cause of massive upper gastrointestinal bleeding. Surgery 1987;102:548-552.

3. Christidou A, Tzathas C, Khuffash O, Triantaphyllou G. Upper gastrointestinal bleeding due to ectopic varices in a patient with alcoholic cirrhosis. Ann Gastroentol 2003;16:179-182.

4. Kotfila R, Trudeau W. Extraesophageal varices. Dig Dis 1998;16:232241.

5. Itzchak Y, Glickman MG. Duodenal varices in extrahepatic portal obstruction. Radiology 1977;124:619-624.

6. Tsuji H, Okano H, Fujino H, et al. A case of endoscopic injection sclerotherapy for a bleeding duodenal varix. Gastroenterol Jpn 1989; 24:60-64.

7. Sans M, Llach J, Bordas JM, et al. Thrombin and ethanolamine injection therapy in arresting uncontrolled bleeding from duodenal varices. Endoscopy 1996;28:403.

8. Liu Y, Yang J, Wang J, et al. Clinical characteristics and endoscopic treatment with cyanoacrylate injection in patients with duodenal varices. Scand J Gastroenterol 2009;44:1012-1016.

9. Yoshida Y, Imai Y, Nishikawa M, et al. Successful endoscopic injection sclerotherapy with N-butyl-2-cyanoacrylate following the recurrence of bleeding soon after endoscopic ligation for ruptured duodenal varices. Am J Gastroenterol 1997;92:1227-1229.

10. Yoon YJ, Hahn KH, Lee SY, et al. A case of massive bleeding duodenal varices successfully controlled with transjugular intrahepatic portosystemic shung. Korean J Gastroenterol 2001;38:292-295.

11. Ohta M, Yasumori K, Saku M, Saitsu H, Muranaka T, Yoshida K. Successful treatment of bleeding duodenal varices by balloon-occluded retrograde transvenous obliteration: a transjugular venous approach. Surgery 1999;126:581-583.

12. Ponec RJ, Kowdley KV. Paradoxical cerebral emboli after transjugular intrahepatic portosystemic shunt and coil embolization for treatment of duodenal varices. Am J Gastroenterol 1997;92:1372-1373.

13. Shin EJ, Ko CW, Magno P, et al. Comparative study of endoscopic clips: duration of attachment at the site of clip application. Gastrointest Endosc 2007;66:757-761.

14. Yol S, Belviranli M, Toprak S, Kartal A. Endoscopic clipping versus band ligation in the management of bleeding esophageal varices. Surg Endosc 2003;17:38-42.

15. Morimoto T, Fu KI, Konuma H, et al. Endoscopic hemostasis with hemoclips for active gastric variceal bleeding. Endoscopy 2010;42 Suppl 2:E333-E334. 